\title{
LES LABYRINTHES DU POUVOIR : LES MAIRIES DU CHANGEMENT FACE AUX ADMINISTRATIONS
}

\author{
Rodrigo Blanca, Ernesto Ganuza, Traduction par Irène Jami
}

\author{
La Découverte | «Mouvements»
}

2018/2 nº 94 | pages 36 à 44

ISSN 1291-6412

ISBN 9782348036125

Article disponible en ligne à l'adresse :

https://www.cairn.info/revue-mouvements-2018-2-page-36.htm

Distribution électronique Cairn.info pour La Découverte.

(C) La Découverte. Tous droits réservés pour tous pays.

La reproduction ou représentation de cet article, notamment par photocopie, n'est autorisée que dans les limites des conditions générales d'utilisation du site ou, le cas échéant, des conditions générales de la licence souscrite par votre établissement. Toute autre reproduction ou représentation, en tout ou partie, sous quelque forme et de quelque manière que ce soit, est interdite sauf accord préalable et écrit de l'éditeur, en dehors des cas prévus par la législation en vigueur en France. Il est précisé que son stockage dans une base de données est également interdit. 


\section{Les labyrinthes du pouvoir : les mairies du changement face aux administrations}

\author{
Par Rodrigo \\ BLANCA* \\ et ERNesto \\ GANUZA** \\ TRADUCTION \\ PAR IRÈnE JAMI
}

1. Leur trajectoire professionnelle à tou.te.s est retracée sur la page web de la municipalité de Madrid http://www.madrid.es

* Coordinateur du groupe municipal Ganemos Córdoba, coalition municipale pour le changement qui dirige la ville de Cordoue depuis les élections de 2015.

** Chercheur au Consejo Superior de Investigaciones Científicas (CSIC)/ Instituto de Estudios Sociales Avanzados (IESA), spécialiste des questions de participation.
Madrid, 3,5 millions d'habitant.e.s, épicentre de la quatrième aire métropolitaine d'Europe par sa taille, est aujourd'hui gouvernée par un parti qui n'existait pas avant 2015. Après plus de 25 ans de gouvernements conservateurs, la cité madrilène est passée sous l'autorité de personnes quasiment dépourvues d'expérience de politique institutionnelle. Neuf des onze membres de la Junta de Gobierno Local - l'organe de gestion le plus important des municipalités en Espagne - de Madrid (en février 2018) n'avaient aucune expérience de politique institutionnelle avant 2015. Ce sont en majorité des universitaires, qui étaient activistes ou militant.e.s dans des mouvements sociaux ${ }^{1}$. Cette description photographique du gouvernement madrilène est très proche de celles que l'on peut observer dans d'autres villes comme Barcelone, dont la maire était jusqu'en 2013 la porte-parole du mouvement de la plateforme des victimes du crédit hypothécaire (PAH, Plataforma de Afectados por la Hipoteca), I'un des mouvements de protestation les plus emblématiques de la décennie dans le pays. On rencontre des situations similaires à La Corogne, Saint-Jacques de Compostelle, Saragosse, Valence ou Cadix. Dans nombre d'autres villes (Cordoue, Alicante, Getafe, Séville..), des élu.e.s issu.e.s de nouveaux partis ont rejoint les groupes parlementaires des conseils municipaux, même lorsque les élections ne leur ont pas donné le pouvoir. Les élections de mai 2015 ont, sans aucun doute, été l'occasion d'affirmer partout, au niveau municipal, une nouvelle rhétorique qui a permis de moduler, avec plus ou moins de succès, la gestion politique de certaines villes.

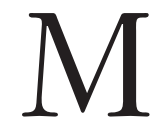

ais quel sens donner à cette irruption politique ? Le discours de ce nouveau municipalisme a toujours mis l'accent sur ses affinités avec les protestations du 15-M. Si les candidatures, lors 
des élections, ne présentaient pas de liens organiques entre elles, les similitudes entre leurs programmes les associaient aux protestations des Indignés. Le "ils ne nous représentent pas " des Indignés qui contestaient les partis politiques en 2011, et le "gouverner autrement" des nouveaux. nouvelles candidat.e.s aux municipales de 2015, se rejoignent dans un récit qui se distancie à l'égard d'une politique élitiste et place la citoyenneté au centre de la gestion ${ }^{2}$. Le 15-M est le lien entre la majorité des candidatures, non tant pour ses résultats matériels, que parce qu'il est parvenu à déplacer le centre de la politique vers un nouveau topos dont le noud est la citoyenneté, partagé par l'opinion publique ${ }^{3}$. Près de $10 \%$ de la population espagnole a participé à un événement du mouvement dans les rues au cours de l'année 2011. Une enquête de Metroscopia-El Pais du mois de juillet de cette année-là montre que $71 \%$ des Espagnol.e.s considéraient le 15-M comme un mouvement pacifique qui régénérait la démocratie (83\% des électeur.trice.s du Parti socialiste ouvrier espagnol, (PSOE, social-démocrate et $54 \%$ des électeur.trices du Parti populaire (PP, libéral-conservateur), contre $17 \%$ qui le considéraient comme un mouvement anti-système. 79 \% des enquêté.e.s affirmaient, de plus, que les manifestations des Indignés étaient justifiées.

Le 15-M a été largement critiqué pour son refus d'investir les institutions politiques ${ }^{4}$. Mais c'est précisément ce renoncement à présenter un programme politique aux élections générales du mois de novembre 2011 qui a permis par la suite l'émergence d'un espace de débat, en marge du mouvement lui-même, sur la possibilité de sauter le pas institutionnel. L'irruption, quelques années plus tard, de ces candidatures aux municipales, peut se comprendre comme une tentative pour imposer ce nouveau topos politique dans les institutions locales, comme celle de Podemos au niveau national'5 . Un nouveau topos qui, tout en renonçant à être le 15-M, y cherchait sa légitimité, et articulerait l'essentiel des programmes politiques autour d'un imaginaire du commun, à l'encontre d'une rhétorique mercantiliste.

Le pas que nombre d'activistes ont franchi vers la politique institutionnelle a fait émerger des questions longtemps discutées entre les spécialistes des mouvements sociaux. Gouverner, ce n'est jamais la même chose que manifester. Il faut passer d'une logique de résistance et de dénonciation à une logique de gestion et de proposition. Les institutions, de plus, présupposent un cadre d'action incontournable, qui oblige les acteurs.actrices politiques à coordonner leur action dans le respect de la loi, en association avec les technicien.ne.s des municipalités, et les expose constamment aux médias alors qu'ils.elles doivent, comme n'importe quel autre parti, appliquer leur programme politique. La recherche
2. A. Fernandez-Savater, "Le 15-M et la crise de la culture consensuelle en Espagne”, Lignes, 39, 2012, p. 148-161.

3. H. Marzolf, E. Ganuza, "Les indignés au pouvoir. Le cas de Podemos en Espagne". Dormira Jamais blog en http://dormirajamais. org/podemos/, 2015.

4. P. Petit, "Réflexions d'un républicain sur le 15-M", La Vie des idées, septembre 2011, http:// www.laviedesidees. fr/Reflexions-d-unrepublicain-sur-le.html

5. H. Nez, Podemos, de l'indignation aux élections, Paris, Les petits matins, 2015.

\section{Gouverner, ce n'est jamais la même chose que manifester. Il faut passer d'une logique de résistance et de dénonciation à une logique de gestion et de proposition.}


de nouveaux équilibres entre les positions les plus en rupture de ces candidatures et la nécessité d'investir les institutions peut sans aucun doute atténuer l'idiosyncrasie des expériences. Mais c'est aussi précisément le moment où on est confronté aux frictions résultant d'un programme politique construit autour d'une rhétorique sur le commun. Il ne faut pas oublier que l'administration publique, en Espagne et plus généralement en Europe, intègre depuis des décennies des outils de gestion du monde privé (New Public Management), si bien qu'un programme politique conçu selon un horizon différent génère de multiples contradictions. Autrement dit, l'efficacité des nouveaux partis ne doit pas seulement être considérée en fonction des résultats obtenus à partir de leurs programmes politiques. Elle doit aussi être replacée dans un processus où sont débattues des questions essentielles, qui portent sur la signification que l'on attribue à la gestion publique. Pour rendre compte de ce problème, nous avons réalisé des entretiens avec des membres des nouvelles listes municipales dans les villes de Cadix, Cordoue, Madrid, SaintJacques de Compostelle et Saragosse.

\section{- Le défi de l'agenda municipaliste : « Nous ne sommes pas de la marchandise entre les mains des politiciens et des banquiers "}

Outre un diagnostic commun de la situation politique (mauvaise gestion économique; classe politique corrompue et isolée de la société), construit par le 15-M, les nouveaux partis se sont présentés aux élections municipales avec un horizon politique dont les trois piliers étaient : 1) la défense des droits fondamentaux et de la dignité de la citoyenneté (éducation, santé, logement), 2) le choix d'un modèle économique social et solidaire et 3) le renforcement de la gestion démocratique des municipalités.

La rhétorique politique du nouveau municipalisme s'est affirmée depuis le début comme une alternative au modèle de gestion publique dominant

La rbétorique politique du nouveau municipalisme s'est affirmée depuis le début comme une alternative au modèle de gestion publique dominant dans les administrations au cours des dernières années. dans les administrations au cours des dernières années. Les nouvelles candidatures étaient opposées à la privatisation des services publics, à une conception du public oblitérée par des critères mercantiles, selon laquelle les citoyen.ne.s sont d'abord des client.e.s, et rejetaient les politiques publiques obéissant à des critères de marché (efficacité économique). Elles proposaient un espace dans lequel le.la citoyen.ne

6. P. DARdot eT C. Laval, Commun. Essai sur la révolution au $X X I^{e}$ siècle, Paris, La Découverte, 2014. aurait une voix active dans les affaires communes et les services publics s'inspireraient d'une logique de commun ${ }^{6}$. Nous prêterons ici attention, non pas tant au type de propositions politiques concrètes faites pour traduire cet horizon, qu'au processus par le biais duquel ces propositions 
ont été formulées dans le but de parvenir à les faire adopter dans les budgets publics, indépendamment de leur succès ou de leur échec.

Cette question pointe l'importance de la dimension culturelle de la proposition portée par les nouveaux partis. Il ne suffit pas de formuler de bonnes propositions, il faut aussi qu'elles soient mises en œuvre, parce qu'il est nécessaire de les situer dans un circuit spécifique, en premier lieu celui de l'administration publique. Cela implique un circuit culturel qui définit les bonnes formes de gestion publique, mais aussi les limites de ce qui est recevable. Les

Contre cette logique mercantile, dans différentes villes, les nouvelles candidatures ont prévu d'introduire des clauses sociales dans les procédures de signature de contrats. difficultés rencontrées pour faire aboutir une proposition donnée ne tiennent pas seulement aux capacités des représentant.e.s, et les échecs ne sont pas imputables à leur manque de volonté. L'administration elle-même obéit à des règles qu'il faut respecter dans le scénario institutionnel, mais les fonctionnaires aussi ont des règles avec lesquelles il faut compter pour mettre en œuvre les mesures politiques. C'est là que la rhétorique politique portée par les nouveaux partis livre sa bataille la plus importante, parce qu'il ne s'agit pas de son caractère opérationnel mais de sa viabilité, qui dépend de sa dimension aussi bien technique que juridique.

Par exemple, l'administration publique, engagée depuis des années dans un processus de modernisation qui soumet la majorité des services publics aux normes du marché, a des difficultés à viabiliser des mesures régulées par d'autres normes. On le constate notamment dans la passation de marchés publics, où les fournisseurs sont constamment incités à un type de compétition caractérisé par l'économie de ressources ou "l'offre économique la plus avantageuse ". Ce critère peut arriver à constituer $50 \%$ ou jusqu'à $75 \%$ de la notation pour l'adjudication d'un contrat spécifique en Espagne. De même, il n'existe pratiquement pas de mécanismes de fiscalisation et de surveillance de l'exécution effective des contrats, si bien que les entreprises commencent par proposer des tarifs délibérément minorés, auxquels s'ajoutent fréquemment des surcoûts qui viennent multiplier le prix final des interventions. Contre cette logique, dans différentes villes, les nouvelles candidatures ont prévu d'introduire des clauses sociales dans les procédures de signature de contrats. Une façon de chercher des alternatives à une offre économique à la baisse, en incitant, au contraire, les fournisseurs à se montrer coopératifs, à intégrer du personnel précaire ou de petites entreprises, au bénéfice direct ou indirect de la ville, des politiques d'insertion ou de l'emploi. Cette mesure se heurte à la conception des relations économiques répandue dans les administrations publiques. D'où une discussion sur la conception de la rentabilité publique et de l'efficacité des politiques. Un des nouveaux élus considère que ces mesures suscitent chez les fonctionnaires des services 
publics des réactions opposées à une logique des communs. Non pas tant parce qu'elles seraient impossibles à mettre en ouvre, que parce qu'elles menaceraient le droit et l'esprit de la libre concurrence sur le marché. L'élu du nouveau parti qui défend l'adoption de clauses sociales au cours d'une réunion à vocation technique, évalue les retombées auxquelles une ville peut s'attendre en investis-

Par le biais de la loi de rationalisation et de durabilité de l'administration locale de 2013, le gouvernement central a limité l'autonomie et les compétences des municipalités dans le but de neutraliser la dette publique. sant dans un centre commercial : "A priori, l'initiative semble bénéfique pour la ville en général. Elle va satisfaire les besoins de certains riverains, attirer de l'investissement, générer des emplois, et procurer des ressources à la municipalité. Maintenant, si on y réfléchit du point de vue de la ville, on s'aperçoit que chaque création d'un poste de travail dans une grande surface commerciale entraîne la suppression de trois emplois dans le petit commerce de quartier, que cela augmente les risques de contamination liés aux déplacements effectués pour réaliser des achats qu'on pouvait auparavant faire en ville et que cela accroît les déchets générés par la vente de produits qui multiplient les emballages. De plus, $80 \%$ de l'argent dépensé en grande surface sort de la ville et favorise ce qui met les producteurs en difficulté ". Le coordinateur des contrats publics de la municipalité réfute cette argumentation au nom des problèmes que créerait, au détriment de la compétence, l'application de clauses sociales contradictoires avec ce qu'il considère comme la norme fondamentale de la passation de contrats : "Celui qui propose le prix le moins élevé gagne ". Il ne s'agit pas d'une simple divergence, mais d'obstacles réels à la mise en œuvre de moyens politiques aux conséquences concrètes très différentes.

\section{- Le contexte municipal}

Pour comprendre les interactions entre la nouvelle rhétorique et la culture administrative, il nous paraît nécessaire de connaître la structure ou la table de jeu qui sert de cadre aux politiques municipales. En premier lieu, les municipalités dans l'État espagnol sont une administration dont le poids est faible par comparaison avec l'environnement européen. Les impôts locaux espagnols représentaient en $20156 \%$ du PIB contre $11,1 \%$ dans les pays de l'Union européenne. Cette différence s'est accentuée depuis le début de la crise de 2008, entraînant un déclin considérable

7. Rapport final de la commission d'experts pour la révision du modèle de financement local, juillet 2017 , p. 104 . des dépenses locales en Espagne (-7,7\% en termes de PIB) ${ }^{7}$. En second lieu, par le biais de la loi de rationalisation et de durabilité de l'administration locale de 2013, le gouvernement central a limité l'autonomie et les compétences des municipalités dans le but de neutraliser la dette publique. Cette réforme a réduit les pouvoirs locaux des municipalités et les a soumises à de nouvelles formes de tutelle, diminuant la capacité de gestion 
des administrations locales ${ }^{8}$. Par comparaison avec les municipalités dans le contexte européen, par exemple, les dépenses des municipalités espagnoles pour la protection sociale, la santé et l'éducation ont représenté en 2015 0,8 \% du PIB contre une moyenne de 6,1 \% dans l'UE'. Il s'agit donc d'une administration qui dispose d'une marge de manœuvre relativement faible, où une grande part du budget et de la force de travail est déjà engagée dans le maintien de l'environnement, et où la mise en œuvre de nouveaux programmes est perçue comme " une tâche supplémentaire " par des équipes de travail déjà largement saturées. Un troisième aspect à souligner est l'insuffisance de personnel résultant du taux de remplacement ${ }^{10}$, qui a pour effet de limiter les possibilités de nouveaux recrutements dans l'administration publique. Cette mesure, imposée par le gouvernement central depuis 2009, a entraîné une réduction des effectifs de 16,1\% (soit presque 100000 personnes) dans les collectivités locales entre janvier 2010 et janvier $2017^{11}$, débouchant sur un vieillissement non moins significatif de celles-ci $(65,7 \% \text { de plus de } 50 \text { ans })^{12}$. Enfin, il est important de souligner aussi les contraintes financières. La réglementation des dépenses, introduite en 2012 en application de la réforme de l'article 135 de la Constitution ${ }^{13}$, interdit que les dépenses des collectivités locales puissent croitre chaque année davantage que le taux de référence de croissance à moyen terme du PIB. Elle prend comme référence les dépenses réelles de l'année précédente, dans des collectivités en difficulté pour exécuter leur budget, essentiellement en raison du manque de personnel et des "constants changements des règles du jeu " concernant la gestion et l'administration des ressources des impôts locaux. C'est donc un impératif éminemment politique, soumis à des normes économiques, qui interdit la mise en ouvre de budgets expansifs, et qui explique en partie les reliquats de trésorerie des municipalités, d'un montant approximatif de 5000 millions d'euros chaque année, en partie utilisés pour le paiement de la dette. Ce que le président de la Fédération espagnole des municipalités et provinces, engagée dans un combat acharné contre le ministère des finances, formulait ainsi dans sa déclaration du 20 février 2018 : "Notre situation financière et budgétaire s'est détériorée, considérablement détériorée, depuis le début de la mandature locale actuelle, il y a un peu moins de trois ans. Au point que nous ne devons pas hésiter à qualifier notre situation d'insupportable, intenable "14.

\section{- Pratiquer l'innovation sociale dans le labyrinthe de I'hégémonie néolibérale}

Ce contexte de fortes contraintes économiques pèse considérablement sur la capacité des nouveaux partis à proposer des mesures politiques alternatives. Le premier obstacle auquel se heurtent les propositions est l'échelle des décisions: beaucoup dépendent organiquement d'autres administrations territoriales. Par exemple, en Espagne, les municipalités ont besoin de l'accord du gouvernement régional pour modifier les Planes Generales de Ordenación Urbana, documents qui structurent les politiques urbaines de la ville. Ainsi, dans la ville de Cordoue, deux processus
8. La réforme de 2013 élimine de l'article 25.1 la promotion de "toute sorte" d'activités et de services pour la satisfaction des besoins et aspirations de la communauté.

9. Op. cit. note 7 , p. 125 .

10. Le taux de remplacement des effectifs est le ratio qui détermine le nombre de nouveaux recrutements à des emplois publics que peut intégrer une administration publique.

11. Boletín Económico 4/2017, Banco de España, La evolución del empleo en las Administraciones Públicas en la última década, p. 2.

12. Boletín Estadístico del Personal al Servicio de las Administraciones Públicas, Janvier 2017. Ministerio de Hacienda y Función Pública. p. 63.

13. En septembre 2011, la modification de l'article 135 de la Constitution espagnole adoptée par le gouvernement socialiste de José Luis Rodríguez Zapatero avec le soutien du Parti populaire (PP) de Mariano Rajoy, a fait du remboursement de la dette une "priorité absolue ". Cette révision permet de réduire les dépenses d'éducation et de santé, précarisant davantage la situation de la population, dans le seul but de renforcer les garanties aux créanciers de la dette espagnole. (N.d.l.t.)

14. http://www.femp. es/comunicacion/ noticias/montoro-llamaal-presidente-de-lafemp-por-la-reinversiondel-superavit 
15. La Junte d'Andalousie (en espagnol Junta de Andalucía) est l'institution au travers de laquelle s'organise l'autogouvernement de la communauté autonome d'Andalousie, en Espagne (N.d.l.t.). simultanés d'innovation du PGOU ont connu des fortunes très différentes. La Délégation à la santé de la Junte d'Andalousie ${ }^{15}$ a mis moins d'un mois (qui plus est, au mois d'août) pour émettre un avis favorable à la construction d'un centre commercial. En revanche, pour se prononcer sur la possibilité de déplacer en banlieue une cimenterie installée en plein centre urbain, la même Délégation a dépassé le délai de trois mois dont elle disposait pour émettre son avis. Une dynamique que l'on retrouve ailleurs. Les vingt-cinq avis, un an et demi de procédure, du dossier pour la municipalisation du service juridique de Saint-Jacques de Compostelle, ou encore les vingt-neuf que compte déjà le dossier pour la municipalisation du service des plages de Cadix, sont révélateurs à cet égard. Cette attitude discrétionnaire des administrations régionales ou d'État n'est pas imputable à des problèmes d'agenda, mais répond à des critères ad hoc, comme l'a montré la flexibilité du ministère des Finances selon qu'il s'est agi d'approuver les comptes publics de la municipalité de Jaén (gou-
16. En 2017 , le ministère des Finances a placé les comptes de la mairie de Madrid sous tutelle alors que l'équipe municipale d'Ahora Madrid a enregistré d'importants excédents pour l'année 2016, tout en réduisant de quarante pour cent la dette laissée par le gouvernement antérieur du PP. De plus, elle a augmenté l'effort d'investissements de cent deux pour cent, dont soixante quatorze pour cent pour les dépenses sociales. (Ndlt).

17. En particulier la Directive 2014/24 de l'Union européenne qui rend obligatoire l'inclusion de ces clauses dans les contrats. vernée par le PP), ou d'intervenir immédiatement dans le cas de la mairie de Madrid dont les comptes publics, eu égard à la règle même des dépenses du ministère, sont pourtant plus sains ${ }^{16}$.

Mais les obstacles les plus importants pour définir de nouvelles mesures politiques proviennent de l'administration locale elle-même. Les tentatives de certains nouveaux gouvernements municipaux pour intégrer des clauses sociales dans les contrats publics permettent de concevoir nombre des problèmes auxquels se heurte l'institutionnalisation de ce nouveau topos politique. Cette mesure à la portée considérable pourrait avoir des conséquences notables. La passation de contrats publics a représenté $16 \%$ du PIB de l'État espagnol en 2016. De nombreuses municipalités ayant largement privatisé leurs services, les contrats publics peuvent représenter plus du tiers de leurs budgets.

L'introduction de clauses sociales dans les contrats publics privilégie, lors de l'attribution d'un contrat, des critères de qualité et surtout, des critères prenant en compte l'impact social que générerait l'investissement économique. Par exemple, l'application de barèmes qui évaluent les propositions des entreprises en fonction de la précarité des contrats de travail, du respect des accords de branche, ou la résiliation du contrat avec l'entreprise en cas de défaut de paiement de ses salariés. En dépit du fait que les clauses sociales s'appuient sur un support législatif solide (en Espagne et dans l'Union européenne ${ }^{17}$ ), les nouveaux partis ont rencontré de grandes difficultés pour obtenir leur inclusion. Lors de la négociation avec les services techniques municipaux, ces projets se sont heurtés à l'opposition verbale et écrite des services chargés de la passation des contrats, souvent motivée par la défense de la liberté d'entreprise ou par 
leur compétence ou défaut de compétence en matière d'emploi. Si bien que dans de nombreuses villes il a fallu plus de deux ans pour obtenir que les contrats intègrent enfin ces clauses, tandis que dans d'autres la tentative n'a abouti qu'à la marge, ou a même échoué.

Cette résistance "technique" oblige les nouveaux partis à mobiliser des moyens considérables pour élaborer des rapports justifiant la viabilité des dispositions

Au terme de presque trois ans de gouvernements municipaux, c'est désormais au coeur des administrations que le nouveau topos politique livre bataille. proposées. Encore ne parlons-nous ici que de projets qui s'appuient sur la législation européenne. Chaque rapport suscite un contre-rapport qui transforme le travail politique en une constante recherche d'allié.e.s au sein du personnel technique pour fournir un appui administratif et juridique aux projets politiques. Les nouveaux élu.e.s se heurtent à une résistance directe de la part de l'opposition politique, qui peut émaner d'une association d'entrepreneurs ou de gouvernements d'une autre couleur politique à la tête d'une administration à l'échelle supérieure mais, selon un des nouveaux candidats impliqué dans des tâches de gouvernement, " en général [les résistances] sont plus subtiles, plus nuancées, ont beaucoup à voir avec la culture administrative, avec ce que sont la bureaucratie, les organes étatiques... ".

Évoquant les résistances aux nouvelles politiques, beaucoup de candidat.e.s ne mentionnent pas une bataille politique mais culturelle, comme le rapporte ce conseiller de l'un des nouveaux gouvernements municipaux : "Il s'agit d'idéologie au sens large du terme, je ne me réfère pas seulement à droite et gauche, mais ce à quoi on est confronté.e.s ici est l'habitude et la culture d'une organisation. Lorsque, alors que les choses ont toujours été faites d'une certaine façon, arrive un gouvernement qui dit qu'il faut les faire autrement, il est difficile de s'y adapter, et il y a une résistance au changement. À cet égard, c'est aussi perçu comme une correction à ce qui s'est fait depuis toujours ".

Dans leur recherche d'allié.e.s au sein des administrations locales, les nouveaux partis doivent aussi compter avec la façon dont ils.elles sont perçu.e.s par ces mêmes fonctionnaires, prévient un membre du nouveau gouvernement: "Nous sommes de nouveaux.elles arrivant.e.s et beaucoup de fonctionnaires peuvent considérer cela comme un accident temporaire. Autrement dit, ici on a toujours été gouverné.e.s par le PP et le PSOE, maintenant ils.elles sont là pour quatre ans, mais ensuite les choses reprendront leur cours. Alors on ne va pas s'impliquer avec eux. elles parce qu'ensuite, quand les autres reviendront, il y aura des récompenses et des punitions. Cela aussi peut jouer".

\section{- Une course de fond}

Au terme de presque trois ans de gouvernements municipaux, c'est désormais au cœur des administrations que le nouveau topos politique 
livre bataille. Ce qui est en jeu, ce n'est pas la qualité des projets mais l'ouverture, au sein de ces administrations, d'un front culturel. Pour bien des nouveaux.elles responsables politiques, la définition de leur tâche se résume à faire preuve de "constance ", comme si c'était l'élément essentiel pour qu'un projet puisse finalement aboutir, " ils vont finir par te lasser et t'écœurer". Beaucoup s'accordent à dire que le pouvoir est très poreux et qu'il n'est pas possible d'éluder sa dimension administrative. C'est pourquoi il s'avère souvent indispensable de s'appuyer sur des technicien.ne.s compétent.e.s et fiables, "loyaux.ales " à l'égard du gouvernement qui impulse les politiques, comme si la possibilité de gouverner dépendait de ces liens qui ouvrent la voie de la bureaucratie. "Tu peux avoir un programme magnifique, faire de grands discours et avoir toute la volonté politique qu'il faut, mais si tu n'es pas capable de convertir cela en actes administratifs, c'est la mort du gouvernement ". Pour beaucoup des militant.e.s qui se retrouvent aujourd'hui au gouvernement, le pouvoir exige sérénité et combativité, parce que "quand on décide de prendre des mesures politiques qui affectent des intérêts économiques déterminés, on sait que ce ne sera pas facile, qu'on aura les partis de l'ordre contre nous, les moyens de communication contre nous, qu'on aura sûrement aussi la justice contre nous, et bon... il faut l'assumer " . 